\title{
Fisioterapia motora no tratamento do prematuro com doença metabólica óssea
}

\author{
Motor physiotherapy in the treatment of preterm infants with metabolic bone disease
}

\author{
Juliana Moreno ${ }^{1}$, Luciana Volpiano Fernandes², Camila Campos Guerra ${ }^{3}$
}

RESUMO

Objetivo: Revisar o papel da fisioterapia motora no prematuro com risco de desenvolver doença metabólica óssea.

Fontes de dados: Trata-se de uma revisão de literatura publicada entre 1986 e 2009, utilizando as seguintes palavras-chave: prematuro, calcificação fisiológica, modalidades de fisioterapia, doenças ósseas metabólicas e os respectivos descritores no idioma inglês. Foram selecionados 29 artigos científicos, via PubMed e ISI Web, além de um capítulo de livro nacional.

Síntese dos dados: As doenças ósseas metabólicas compreendem um conjunto de condições relacionadas a alterações no processo de calcificação fisiológica, levando desde à fragilidade estrutural até ao desenvolvimento de fraturas. A aplicação rotineira de exercícios de mobilização passiva articular, massagem e posicionamento está relacionada ao ganho ponderal, ao aumento na densidade e no conteúdo mineral ósseo.

Conclusões: A implementação de exercícios de fisioterapia motora parece proporcionar estabilidade ou estímulo para a formação óssea, podendo, consequentemente, prevenir e/ou minimizar as complicações decorrentes da doença metabólica óssea.

Palavras-chave: prematuro; calcificação fisiológica; modalidades de fisioterapia; doenças ósseas metabólicas.

\section{ABSTRACT}

Objective: To review the role of motor physiotherapy in the treatment of preterm infants at risk of developing metabolic bone disease.

Data sources: This is a review of articles published between 1986 and 2009, using the following key-words: premature infant physiologic calcification, physiotherapy techniques, metabolic bone diseases and the respective Portuguese-language descriptors. Twenty nine scientific articles were selected in the PubMed and ISI Web databases, along with one chapter of a Brazilian book.

Data synthesis: Metabolic bone diseases are a set of conditions related to abnormalities in the physiologic calcification process. They lead to problems going from structural frailness to fracture development. Routine application of passive joint mobilization exercises, massage and positioning exercises correlate with weight gain and increasing bone mineral content and density.

Conclusions: Implementation of motor physiotherapy exercises could provide stability or stimulation for bone formation and may consequently avoid or minimize the complications resulting from metabolic bone disease of prematurity.

Key-words: infant, premature; calcification, physiologic; physiotherapy techniques; bone diseases, metabolic.
Instituição: Universidade Federal de São Paulo (Unifesp), São Paulo (SP), Brasil

${ }^{1}$ Especialista em Fisioterapia Respiratória Pediátrica e Neonatal pela Unifesp, São Paulo, SP, Brasil

${ }^{2}$ Pós-graduanda em Pediatria e Ciências Aplicadas à Pediatria do Departamento de Pediatria da Unifesp, São Paulo, SP, Brasil

${ }^{3}$ Especialista em Fisioterapia Respiratória pela Santa Casa de Misericórdia de São Paulo; Coordenadora da Fisioterapia Pediátrica do Hospital das Clínicas Luzia de Pinho Melo, São Paulo, SP, Brasil

\author{
Endereço para correspondência: \\ Juliana Moreno \\ Rua Desembargador Carneiro Ribeiro, 159 - Artur Alvim \\ CEP 03569-000 - São Paulo/SP \\ E-mail: jumoreno15@yahoo.com.br \\ Conflito de interesse: nada a declarar
}

Recebido em: 12/9/2009

Aprovado em: 26/1/2010 


\section{Introdução}

A população de pacientes internados em unidades de cuidados intensivos neonatais modifica-se a cada ano, sendo os avanços tecnológicos e assistenciais responsáveis por reduzir a mortalidade em $15 \%$ na última década, com consequente aumento na sobrevida de prematuros de muito baixo peso ${ }^{(1)}$. Sabe-se, porém, que tais mudanças são acompanhadas de morbidades que afetam diretamente o prognóstico desses recém-nascidos em curto e longo prazo, envolvendo os mais diversos órgãos e sistemas ${ }^{(2,3)}$.

O tecido ósseo sofre consequências deletérias do parto prematuro, visto que o último trimestre de gravidez é o período em que ocorre o maior acréscimo mineral ósseo, com seu pico entre 34 e 36 semanas de gestação ${ }^{(4,5)}$. A deficiência mineral de cálcio e fósforo ${ }^{(5-7)}$, a interrupção do transporte placentário de estrogênio e outros hormônios que promovem formação óssea e a falta de movimentação do feto contra a resistência muscular das paredes do útero (estímulo mecânico e carga ao osso) são fatores envolvidos na fisiopatologia da doença metabólica óssea (DMO) $)^{(8-10)}$.

A DMO é uma condição frequente em prematuros, tendo incidência maior que $60 \%$ quando o peso de nascimento é inferior a $1000 \mathrm{~g}^{(11)}$. Engloba, em sua forma mais leve, a osteopenia da prematuridade e, em estágios mais avançados, a osteomalácea e o raquitismo ${ }^{(9)}$.

O grau do comprometimento depende do estágio da doença: quadros leves geralmente cursam com edemas de articulações costo-condrais; quadros moderados, com redução ou interrupção do crescimento longitudinal do osso; enquanto casos extremos podem provocar fraturas ou atelectasias (por falta de sustentação da caixa torácica). Tais manifestações tornam-se evidentes entre a sexta e a $12^{\mathrm{a}}$ semanas do período pós-natal ${ }^{(5)}$. Além da prematuridade, são citados como fatores relacionados à DMO a nutrição parenteral prolongada (NPP), a icterícia, o atraso na introdução de nutrição enteral ou sua pobreza em conteúdo mineral, a terapia diurética prolongada, a displasia broncopulmonar, além do uso de teofilinas por períodos longos. A administração de corticoides, a enterocolite necrosante e as doenças hepáticas também contribuem para a DMO em prematuros ${ }^{(6,12)}$.

Com a elucidação do curso natural da doença e dos fatores geralmente associados ao seu desenvolvimento, torna-se fundamental que uma equipe multidisciplinar atue na prática clínica para prevenir ou minimizar os déficits sobre a mineralização óssea em prematuros de risco. Tal objetivo é um desafio para os profissionais de saúde, visto que, mesmo diante da administração de dietas especiais com fórmulas fortificadas, o acréscimo mineral no meio extrauterino não se equipara ao esperado no último trimestre de gestação ${ }^{(12)}$.

Diante do risco potencial de desenvolvimento da DMO e suas consequências e da dificuldade em atingir resultados suficientemente satisfatórios com métodos terapêuticos convencionais, a fisioterapia alia-se à terapia nutricional, objetivando minimizar o decréscimo mineral ósseo predominante nas primeiras semanas de vida de crianças prematuras.

Assim, essa revisão aborda métodos seguros para prevenir e tratar o recém-nascido com risco de desenvolver DMO, sendo considerado como terapêutica uma rotina com o posicionamento programado e supervisionado, a massagem e a mobilização passiva de articulações apendiculares. Buscaram-se artigos relevantes para o tema publicados entre 1986 e 2009, utilizando as seguintes palavras-chave: prematuro, calcificação fisiológica, modalidades de fisioterapia, doenças ósseas metabólicas e os respectivos descritores no idioma inglês. Foram selecionados 29 artigos científicos, via MedLine, PubMed e ISI Web, além de um capítulo de livro nacional.

\section{Fisioterapia no recém-nascido com fragilidade óssea}

Não é raro defrontar-se com uma equipe que contraindica a fisioterapia no prematuro com DMO. Importante ressaltar que a assistência fisioterapêutica em neonatologia não se resume à aplicação de manobras de higiene brônquica, visto que é dever do profissional atualizado avaliar, acompanhar e tratar o prematuro do ponto de vista motor, identificando precocemente qualquer evidência ou risco para atraso no desenvolvimento neuropsicomotor.

Convém, porém, advertir que, antes da realização dos exercícios propostos, critérios mínimos devem ser analisados para que a assistência possa trazer apenas benefícios. É imprescindível que o recém-nascido tenha idade gestacional de pelo menos 27 semanas, e que no momento do início de intervenção tenha idade pós-natal maior que 72 horas, com a matriz germinativa periventricular, no cérebro, menos suscetível a sangramento. $\mathrm{O}$ paciente precisa estar estável do ponto de vista hemodinâmico, com ganho de peso e em ventilação espontânea (podendo fazer uso de oxigênio). Crianças com diagnóstico de displasia broncopulmonar e sepse neonatal tratada que fizeram uso de diuréticos, corticoesteroides, xantinas ou sedativos e nutrição parenteral podem se beneficiar 
do programa fisioterapêutico por se tratar de grupo de risco para deficiência mineral óssea. Visto que a imobilidade cursa com redução na massa óssea, prematuros com afecção no sistema nervoso central e síndromes genéticas podem ser candidatos à fisioterapia motora ${ }^{(13)}$. De modo geral, faz-se necessário o consentimento da equipe médica e a discussão da importância assistencial em cada caso.

As condutas revistas neste artigo devem ser suspensas na presença de hipertensão ou hipotensão arterial, taquicardia ou bradicardia, taquipneia ou apneia, desconforto respiratório (uso da musculatura acessória, batimento de asa do nariz, tiragens intercostal e subdiafragmática, retração de fúrcula e xifoidea, respiração paradoxal e/ou cianose), saturação periférica de oxigênio menor do que $88 \%$ e perda de peso. A avaliação da dor por meio de escalas validadas também direciona a interrupção dos exercícios ${ }^{(14)}$.

A escolha do método terapêutico é individual e deve respeitar a evolução e o grau de fragilidade óssea de cada prematuro, podendo ou não ser eleita apenas uma modalidade de fisioterapia.

\section{Posicionamento}

A tensão gerada ao osso favorece um mecanismo regulatório conhecido por mechanostat, em que a formação óssea é diretamente proporcional ao grau de tensão e carga mecânica fornecida ao sistema esquelético ${ }^{(10)}$. Sabe-se que a movimentação ativa influencia diretamente a formação da massa óssea; assim, lesões cerebrais que cursam com alteração no tônus muscular e redução dos movimentos antigravitários são fatores predisponentes ao desenvolvimento de $\mathrm{DMO}^{(15)}$. Sugere-se que o emprego de determinadas práticas (comportamentais e ambientais), como a imobilização e o uso de fraldas, pode privar os prematuros de estímulo mecânico e interfere, de alguma forma, na movimentação ativa ${ }^{(16)}$.

É controverso se a preferência por determinada postura pode influenciar o desenvolvimento motor de lactentes saudáveis, porém, é indiscutível que o emprego de posicionamentos fixos e assimétricos em populações de risco culmina em encurtamentos e deformidades em longo prazo ${ }^{(17,18)}$.

Prematuros posicionados em pronação continuamente apresentam importantes alterações motoras, como: desequilíbrio entre os grupos flexores e extensores do pescoço; maior incidência de opistótono; exagerada extensão do tronco que restringe a movimentação centrípeta anterior; dificuldade em permanecer na postura lateral; tendência a manter membros superiores flexionados e abduzidos; cabeça com rotação para um dos lados; predisposição a desvio do pé; e encurtamentos musculares com abdução e rotação externa dos músculos do ombro. A consequência é o bloqueio para a movimentação ativa, gerando adaptação para movimentos compensados e não funcionais. Com esse fundamento, sugere-se uma rotatividade programada quanto ao posicionamento dos prematuros estáveis do ponto de vista hemodinâmico. A alternância postural a cada três horas parece evitar disfunções tônicas em prematuros ${ }^{(19)}$ e, consequentemente, a amplitude plena dos movimentos permite o desenvolvimento fisiológico para o tecido ósseo.

Dentre os diversos benefícios, a alternância de postura fornece estímulo mecânico que proporciona um desenvolvimento mais sinérgico da musculatura, permitindo tônus mais adequado, amplitude de movimento mais ampla e, consequentemente, maior movimentação ativa ${ }^{(20)}$.

Na população de prematuros com maior fragilidade óssea, porém estáveis, o posicionamento alternado é um auxílio que não oferece risco, desde que realizado com devidos cuidados e por pessoal capacitado.

\section{Massagem}

Diversos estudos em neonatologia enfatizam benefícios da massagem em prematuros, abrangendo relaxamento, ganho de peso, aumento significativo nos níveis de cálcio sérico, do propeptídeo carboxiterminal do pró-colágeno tipo I (PICP sérico - marcador de formação óssea) e do paratormônio ${ }^{(16,21,22)}$. Acredita-se que a massagem possa ser realizada em prematuros estáveis e com peso mínimo de $600 \mathrm{~g}$ ao nascimento.

Sugere-se um protocolo de massagem composto por compressões no dorso do bebê, que deve inicialmente estar posicionado em pronação. Deve-se respeitar uma sequência sem causar estresse ou estímulo excessivo ao neonato, sendo: da face e cabeça da criança até o pescoço; do pescoço aos ombros; da região superior do dorso à cintura; da coxa aos pés (em ambos os membros inferiores); dos ombros às mãos e vice-versa (em ambos os membros superiores). Cada região deve ser pressionada durante um minuto, com uma frequência a cada cinco segundos ${ }^{(16)}$. A mudança para decúbito dorsal também pode ser realizada, sendo que benefícios podem ser conquistados pela massagem nessa postura ${ }^{(21)}$. Recomenda-se pressão moderada durante a massagem, pois esta favorece a acentuação da atividade vagal. A massagem promove relaxamento à criança, gerando menor gasto energético e favorecendo o ganho de peso ${ }^{(12,21)}$. 


\section{Mobilização passiva articular}

Programas de estimulação motora em Unidade de Terapia Intensiva (UTI) vêm sendo aplicados há mais de duas décadas, porém, somente em 1995 a atuação fisioterapêutica em prematuros com risco para desenvolvimento de DMO passou a ser revista, indicando que exercícios de mobilização passiva interferem favoravelmente na mineralização óssea ${ }^{(23)}$. O protocolo original baseia-se em exercícios de flexo-extensão somados à leve compressão, sendo cada articulação do esqueleto apendicular movimentada cinco vezes. Com uma sessão diária durante quatro semanas (dois dias de repouso na semana), prematuros com pelo menos duas semanas de vida conquistaram maior ganho de peso, diferença quanto à densidade e à massa óssea e melhora no conteúdo mineral ósseo $(\mathrm{CMO})$ em relação ao grupo controle ${ }^{(24)}$. Tais exercícios demonstram outros benefícios além do ganho de peso, como maior comprimento do antebraço, maior área óssea (avaliado por DEXA - dual photon absorptiometry), maior CMO e massa magra ${ }^{(25)}$.

Quando o protocolo é aplicado mais tardiamente, por volta da quarta semana de vida, também se observa aumento significativo do peso corporal, melhora na mineralização óssea, com aumento de marcadores de formação óssea (fosfatase alcalina e PICP), além de redução do telopeptídeo carboxiterminal (ICTP), considerado como marcador de reabsorção óssea e como reflexo da atividade osteoclástica. Nenhum exame de imagem confirma a relação desses marcadores com a real densidade óssea ${ }^{(26)}$.

Se há estabilidade clínica e condições para realizar a fisioterapia motora, prematuros que, entre o quarto e o sétimo dias de vida sejam adequados para a idade gestacional, estejam com nutrição enteral apropriada e peso maior que $800 \mathrm{~g}$ podem ser submetidos aos exercícios. Após quatro semanas de intervenção, à análise do ultrassom quantitativo pode-se observar estabilização na velocidade do som, sendo a mesma reduzida em grupos que não recebem mobilização passiva articular (a velocidade do som é diretamente proporcional à densidade e $\mathrm{CMO})^{(8,27)}$.

O prolongamento da aplicação diária de mobilização passiva para oito semanas fornece uma chave importante sobre o período necessário de realização dos exercícios para se obter benefícios na mineralização óssea. A ausência de alteração na massa óssea em prematuros submetidos à flexo-extensão passiva é benéfica, uma vez que quando grupos não submetidos aos exercícios têm redução de massa óssea. O período de maior atenuação da perda de massa óssea ocorre até a quarta semana de intervenção, porém, a ampliação para oito semanas permite observar que os resultados até então obtidos se mantiveram durante esse período, sugerindo a importância de aplicar o protocolo por um tempo prolongado ${ }^{(4)}$.

Durante as primeiras oito semanas de vida, sabe-se que prematuros demonstram aumento espontâneo de marcadores de formação óssea, como a fosfatase alcalina, e redução de marcadores de reabsorção, como o ICTP. Entretanto, não há associação comprovada entre os marcadores ósseos e a velocidade do som ao examinar o recém-nascido com ultrassom quantitativo. Tal dado permanece ainda obscuro, porém, sugere-se a possibilidade de que as mudanças nos marcadores precedam mudanças estruturais ou na densidade óssea. Todavia, resultados opostos também foram encontrados, com mudanças no exame de ultrassom e ausência de alterações dos marcadores ${ }^{(4,27,28)}$.

Recentemente, a Biblioteca Cochrane publicou uma revisão sistemática sobre os efeitos dos programas de atividade física no crescimento e mineralização óssea em prematuros. A revisão incluiu apenas os artigos que utilizaram exercícios de mobilização passiva. Nenhum risco potencial foi demonstrado pela revisão, e não foram notificados episódios de fratura. Os revisores mencionam a pobre metodologia dos estudos e concluem que há fraca evidência de que exercícios de mobilização passiva promovam ganho de peso e mineralização óssea em curto prazo em bebês prematuros, sendo sua importância clínica questionável. Entretanto, resultados significativos foram encontrados nos artigos inseridos nesta revisão ${ }^{(29)}$.

A idade do recém nascido parece exercer influência direta sobre o CMO, visto que prematuros com IG média ao nascimento de 29 semanas, avaliados com idade pós-conceptual de 40 semanas, apresentam menor $\mathrm{CMO}$ (40\%), comparados aos nascidos a termo (RNT). Porém, quando os prematuros atingem uma média 50 semanas de idade pós-conceptual, observa-se um valor cinco a dez vezes maior de CMO do que no grupo de RNT. Dessa forma, acredita-se que a discrepância da mineralização óssea entre prematuros e recém-nascidos a termo seja amenizada quando os primeiros atingem aproximadamente 40 semanas de idade pós-conceptual. Todavia, é importante ressaltar que tal resultado foi encontrado em uma amostra de recém-nascidos adequados para a idade gestacional, estando o peso provavelmente relacionado ao resultado obtido ${ }^{(30)}$.

Diante da variabilidade da metodologia dos estudos (exames utilizados, peso ao nascimento, amostra reduzida e interferência de cuidados gerais como estímulo tátil no grupo controle, entre outros), os resultados encontrados 
podem ter sido subestimados ou superestimados, dificultando a elucidação dos efeitos da atividade física em prematuros com risco de DMO. Apesar da divergência dos estudos, a maioria indica benefícios da mobilização passiva articular no prematuro, inclusive quando os exercícios são realizados antes de 40 semanas de idade gestacional corrigida e por períodos prolongados ${ }^{(4,27)}$.

\section{Considerações finais}

Confiar na resolução espontânea da mineralização óssea em uma população de risco parece não ser o ideal, visto que os fatores de risco podem se sobrepor, aumentando a fragilidade óssea e todas as suas consequências deletérias. É fundamental que o fisioterapeuta tenha experiência e conhecimento para decidir qual método pode aliar benefício e segurança para o paciente, além de estabelecer junto à equipe médica o melhor momento para iniciar a terapia, e se a mesma deve ser interrompida. Padronizar o atendimento de fisioterapia motora ao prematuro de risco para DMO é importante em uma população de risco para complicações que podem gerar prejuízo na recuperação da doença de base, com o desenvolvimento de sequelas que exigem maior período de internação e maiores custos.

\section{Referências bibliográficas}

1. Horbar JD, Badger GJ, Carpenter JH, Fanaroff AA, Kilpatrick S, LaCorte M et al. Trends in mortality and morbidity for very low birth weight infants, 19911999. Pediatrics 2002;110:143-51.

2. Chan K, Ohlsson A, Synnes A, Lee DS, Chien LY, Lee SK. Survival, morbidity, and resource use of infants of 25 weeks' gestational age or less. Am J Obstet Gynecol 2001;185:220-6.

3. Euser AM, de Wit CC, Finken MJ, Rijken M, Wit JM. Growth of preterm born children. Horm Res 2008;70:319-28.

4. Litmanovitz I, Dolfin T, Arnon S, Regev RH, Nemet D, Eliakim A. Assisted exercise and bone strength in preterm infants. Calcif Tissue Int 2007;80:39-43.

5. Catache M, Leone CR. Critical analysis of pathophysiological, diagnostic and therapeutic aspects of metabolic bone disease in very low birth weight infants. J Pediatr (Rio J) 2001;77(Suppl 1):S53-62.

6. Goulart AL. Osteopenia da prematuridade. In: Kopelman BI, Santos AM, Goulart AL, Almeida MF, Miyoshi MH, Guinsburg R. Diagnóstico e tratamento em neonatologia. São Paulo: Atheneu; 2004. p.393-8.

7. Abrams SA. In utero physiology: role in nutrient delivery and fetal development for calcium, phosphorus, and vitamin D. Am J Clin Nutr 2007;85:604S-7S.

8. McDevitt $\mathrm{H}$, Ahmed SF. Quantitative ultrasound assessment of bone health in the neonate. Neonatology 2007;91:2-11.

9. Rauch F, Schoenau E. Skeletal development in premature infants: a review of bone physiology beyond nutritional aspects. Arch Dis Child Fetal Neonatal Ed 2002;86:F82-5.

10. Sharp M. Bone disease of prematurity. Early Hum Dev 2007;83:653-8.

11. Avila-Díaz M, Flores-Huerta S, Martínez-Muñiz I, Amato D. Increments in whole body bone mineral content associated with weight and length in pre-term and full-term infants during the first 6 months of life. Arch Med Res 2001;32:288-92.

12. Field T, Diego MA, Hernandez-Reif M, Deeds O, Figuereido B. Moderate versus light pressure massage therapy leads to greater weight gain in preterm infants. Infant Behav Dev 2006;29:574-8.

13. Rohana J, Hasmawati J, Zulkifli SZ. Risk factors associated with low bone mineral content in very low birth weight infants. Singapore Med J 2007;48: 191-4.

14. Blauer T, Gerstmann D. A simultaneous comparison of three neonatal pain scales during common NICU procedures. Clin J Pain 1998;14:39-47.

15. Eliakim A, Nemet D, Friedland O, Dolfin T, Regev RH. Spontaneous activity in premature infants affects bone strength. J Perinatol 2002;22:650-2.

16. Aly H, Moustafa MF, Hassanein SM, Massaro AN, Amer HA, Patel K. Physical activity combined with massage improves bone mineralization in premature infants: a randomized trial. J Perinatol 2004;24:305-9.

17. Carmeli E, Marmur R, Cohen A, Tirosh E. Preferred sleep position and gross motor achievement in early infancy. Eur J Pediatr 2009;168:711-5.

18. Porter D, Michael S, Kirkwood C. Is there a relationship between preferred posture and positioning in early life and the direction of subsequent asymmetrical postural deformity in non ambulant people with cerebral palsy? Child Care, Health Dev 2008;34:635-41.

19. Vaivre-Douret L, Ennouri K, Jrad I, Garrec C, Papiernik E. Effect of positioning on the incidence of abnormalities of muscle tone in low-risk, preterm infants. Eur J Paediatr Neurol 2004;8:21-34.

20. Sweeney JK, Gutierrez T. Musculoskeletal implications of preterm infant positioning in the NICU. J Perinat Neonatal Nurs 2002;16:58-70.

21. Ferber SG, Kuint J, Weller A, Feldman R, Dollberg S, Arbel E et al. Massage therapy by mothers and trained professionals enhances weight gain in preterm infants. Early Hum Dev 2002;67:37-45.

22. Vickers A, Ohlsson A, Lacy JB, Horsley A. Massage for promoting growth and development of preterm and/or low birth-weight infants. Cochrane Database Syst Rev 2004;(2)CD000390.

23. Field TM, Schanberg SM, Scafidi F, Bauer CR, Vega-Lahr N, Garcia R et al. Tactile/kinesthetic stimulation effects on preterm neonates. Pediatrics 1986;77:654-8.

24. Moyer-Mileur L, Luetkemeier M, Boomer L, Chan GM. Effect of physical activity on bone mineralization in premature infants. J Pediatr 1995;127:620-5.

25. Moyer-Mileur LJ, Brunstetter V, McNaught TP, Gill G, Chan GM. Daily physical activity program increases bone mineralization and growth in preterm very low birth weight infants. Pediatrics 2000;106:1088-92.

26. Nemet D, Dolfin T, Litmanowitz I, Shainkin-Kestenbaum R, Lis M, Eliakim A. Evidence for exercise-induced bone formation in premature infants. Int $\mathrm{J}$ Sports Med 2002;23:82-5.

27. Litmanovitz I, Dolfin T, Friedland O, Arnon S, Regev R, Shainkin-Kestenbaum $\mathrm{R}$ et al. Early physical activity intervention prevents decrease of bone strength in very low birth weight infants. Pediatrics 2003;112:15-9.

28. Litmanovitz I, Dolfin T, Regev R, Arnon S, Friedland O, Shainkin-Kestenbaum $R$ et al. Bone turnover markers and bone strength during the first weeks of life in very low birth weight premature infants. J Perinat Med 2004;32:58-61.

29. Schulzke SM, Trachsel D, Patole SK. Physical activity programs for promoting bone mineralization and growth in preterm infants. Cochrane Database Syst Rev. 2007;(2):CD005387.

30. Congdon PJ, Horsman A, Ryan SW, Truscott JG, Durward H. Spontaneous resolution of bone mineral depletion in preterm infants. Arch Dis Child 1990;65:1038-42. 